\title{
Pump-Probe Electron Gas Diffraction - Reconstruction of One Molecule From Many
}

\author{
J.C.H.Spence*, R. Kirian* , U. Weierstall* , H.C. Poon** and D. K. Saldin,** \\ * Department of Physics, Arizona State University, Tempe, Az. 85287-1505. \\ ** Department of Physics, Univerisity of Wisconsin, PO Box 413 Milwaukee, WI 53211.
}

The advent of femtosecond lasers which can by used to control X-ray and electron sources promises the development of diffaction techniques for the determination of excited-state structures and time-resolved "movies" of molecular transformations [1, 2]. A recent electron gun design [3] offers $30 \mathrm{fs}, 0.1 \mathrm{pC}$ pulses from $100 \mathrm{kV}$ electrons with a spot size of 500 microns and $3 \mathrm{~nm}$ coherence width. This gun is based on a photocathode, two magnetic lenses, and an RF cavity. In this paper we propose the use of this gun in a pump-probe arrangement, for gas diffraction in combination with a new method of molecular image reconstruction [4,5].

Figure 1 shows the experimental arrangement. Gas-phase molecules are injected using a freeexpansion nozzle at A, with rotational temperatures of a few degrees Kelvin. An Omega filter passes only those electrons which have not lost more than about one electron-volt energy while traversing the gas jet. CCD detectors with high-speed readout have recently become available, and an electron scintillator screen of corresponding speed will be required. The laser beam is split to arrive first at the sample, where intermediate states of the molecules are excited, then, after a delay, to the photocathode, to generate a pulse of electrons which generates a diffraction pattern. The repetition rate is synchronized to the detector. Many patterns may be collected for each delay. Each different delay provides a different frame of the final movie. Since only a small fraction of molecules are excited, differences between patterns from dark and illuminated molecules must be used. We note:

1. The thousand-fold increase in scattering cross-section over X-ray diffraction produces a strong signal, yet multiple scattering effects are negligible through use of $100 \mathrm{kV}$ electrons

2. The low rotational temperature of the gas molecules produces diffraction from static structures, allowing longer pulses containing more electrons.

3. The transverse spatial coherence is adequate for molecular diffraction, preventing interference between electrons scattered by different molecules (as required by the inversion algorithm [4]).

4. The Omega filter removes inelastic scattering, which otherwise dominates for light elements.

5. By working with molecules whose ground state structure is known, the inversion problem for reconstructing the excited states becomes a problem of linear algebra..

The remarkable inversion algorithm (a development of work by Kam [6] ) uses many patterns, each from many identical molecules lying in random orientations, and reconstructs from them the pattern from a single molecule. This can then be phased using iterative phasing methods [7]. In brief, measurable angular correlation functions of diffraction patterns from identical particles are independent of the orientations of the particles. Oversampled diffraction patterns of individual particles (e.g. molecules) may be extracted from these correlation functions, which may then be inverted to yield the molecular potential maps [5]. Figure 2 shows a two-dimensional example of single-particle diffraction pattern recovery for the K-channel protein, using simulated data for molecules aligned along a single axis. Experimental results using X-rays are given in [5].

References

[1] R. Srinivasan et al Helv. Chim. Acta 86, 1761 (2003)

[2] J. Dwyer et al Phil Trans. A364,741 (2006); H. Chapman Nature Materials 8, 299 (2009). 
[3] T. van Oudheusden et al J. Appl Phys. 102, 093501 (2007).

[4] D.K.Saldin, V.L.Shneerson, R. Fung and A. Ourmazd. J. Phys. Cond Matt 21, 134014 (2009)

[5] D.K. Saldin et al. New Journal of Physics, in press (2010).

[6]. Z. Kam, Macromolecules 10, 927 (1977).

[7] G. Ozlanyi et al. Acta Cryst. A61, 147 (2005). S. Marchesini Rev. Sci. Instr. 78, 049901 (2007)

[8] Work supported by NNSA. We are grateful to Prof E. Bauer for useful discussions.

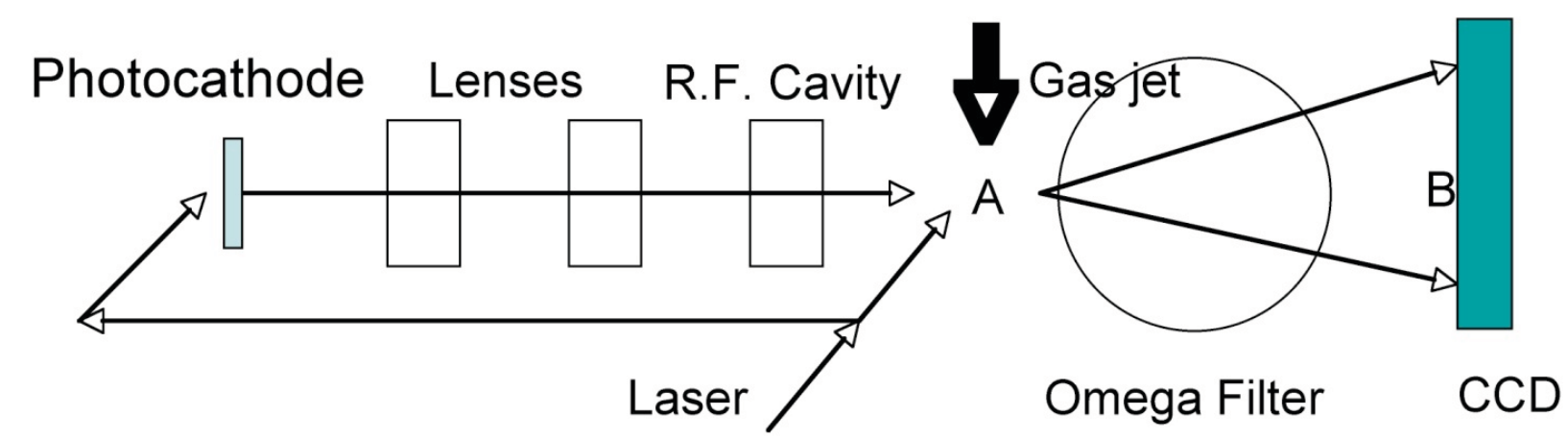

Figure 1. Schematic arrangement for gas electron diffraction. At B a fast-readout CCD operating at 20 $\mathrm{HZ}$ is sychronized with the photocathode. A free-expansion nozzle at A injects gas at low temperature.

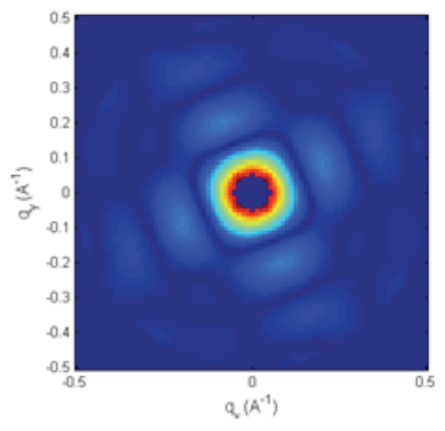

\section{Model DP}

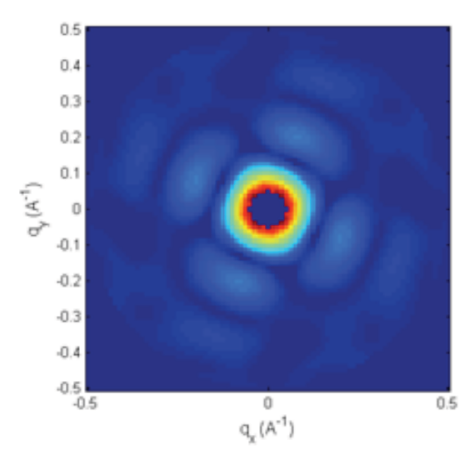

1 particle, 100 DPs

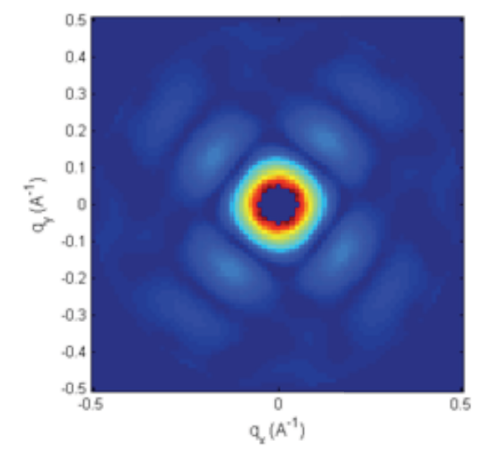

10 particles, 100 DPs

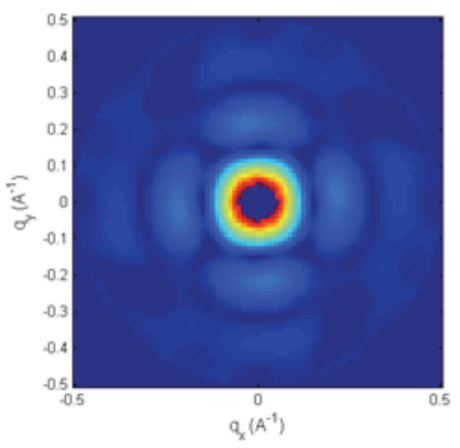

10 particles, 1000 DPs

Figure 2. Simulated examples of the reconstruction of a single-molecule diffraction pattern using the scattering from many identical molecules in random orientations. The number of particles contributing to each pattern, and the number of patterns, is indicated below. The molecules differ by random rotations about a single axis. The random starting phases produce random rotations of the result. Iterative phasing methods can then be used to recover a potential map of the molecule. 